\title{
EXAMPLES OF NON-GAUSSIAN QUASI-INVARIANT DISTRIBUTIONS IN HILBERT SPACE
}

\author{
BY \\ JACOB FELDMAN( ${ }^{(1)}$
}

1. Introduction. Distributions on infinite-dimensional real linear spaces were studied in [5] by I. E. Segal, in connection with quantum statistics. The quasi-invariant (Definition 4) distributions in real Hilbert space can be used to get representations of the commutation relations for dynamical systems with infinitely many variables, as described in [6]. Irreducible representations are obtained if and only if the distribution is ergodic, in the sense of Definition 5, and equivalent representations are obtained if and only if the representations are equivalent in the sense of absolute continuity, Definition 2. Certain known ergodic quasi-invariant distributions are the Gaussian ones. In the present paper are constructed classes of distributions which are not equivalent, in the above sense, to Gaussian ones. This can be done quite easily if one does not require that the distribution be ergodic (see Example 1 in \$3), and with more difficulty if ergodicity is demanded (Example 2).

The author is indebted to L. LeCam for referring him to the theorem used to get Lemma 6.

2. Definitions and general remarks. Let $S$ be a set, $S$ a Borel field of subsets. For some probability measure $\sigma$ on $\delta$, let $\mathbf{A}$ denote the algebra of $\delta$ measurable real-valued functions on $S$, modulo $\sigma$-null functions. After [4], we call such equivalence classes measuroids. Observe that there is an identification between the nonnegative countably additive functions on the idempotents of $\mathbf{A}$ which send the identity to 1 and vanish on no nonzero idempotent, and the probability measures on $S$ which are equivalent to $\sigma$. Such measures we then call suitable to $\mathbf{A}$. An algebra such as $\mathbf{A}$ we call a measuroid algebra. Observe that $\mathbf{A}$ comes equipped with a topology, induced by almost everywhere convergence of functions; and this topology is independent of the particular $(S, \delta, \sigma)$ from which $\mathbf{A}$ arose. Indeed, it can be described purely in terms of the ordering in A. For details, see [4].

Definition 1. A predistribution is a linear map $\bar{m}$ from a real linear space $X$ to a measuroid algebra $\mathbf{A}$ which generates $\mathbf{A}$, in the sense that the polynomials in $\{\bar{m}(x) \mid x \in X\}$ are dense in $\mathbf{A}$. A distribution $m$ is a predistribution $\bar{m}$ together with a choice of a suitable measure for $\mathbf{A}$.

Thus a predistribution induces, in general, many distributions.

Presented to the Society, January 29, 1960 under the title Some examples of quasi-invariant distributions on Hilbert space; received by the editors August 28, 1959.

( $\left.{ }^{1}\right)$ This research was sponsored in part by the Office of Naval Research under Contract Nonr-222(60). 
Definition 2. Two predistributions $\bar{m}: X \rightarrow \mathbf{A}$ and $\bar{m}^{\prime}: X \rightarrow \mathbf{A}^{\prime}$ will be called equivalent, symbolically $\bar{m} \sim \bar{m}^{\prime}$, if there is an algebraic isomorphism $\phi$ from $\mathbf{A}$ onto $\mathbf{A}^{\prime}$ such that $\phi(\bar{m}(x))=\bar{m}^{\prime}(x)$ for all $x \in X$. Two distributions will be called mutually absolutely continuous, symbolically $m \sim m^{\prime}$, if their predistributions are equivalent.

It should be noted that $\phi$ is then unique, since any isomorphism of measuroid algebras is a homeomorphism, and since $\phi$ is determined on a dense subset.

Definition 3. Two distributions $m, m^{\prime}$ will be called strongly equivalent, symbolically $m \approx m^{\prime}$, if

(1) $m \sim m^{\prime}$,

(2) the $\phi$ inducing the correspondence (1) enjoys also the following property: for each bounded Baire function $f$ of $k$ real variables and each $A_{1}, \cdots$, $A_{k} \in \mathrm{A}$, we have $\int f\left(A_{1}, \cdots, A_{k}\right) d \sigma=\int f\left(\phi\left(A_{1}\right), \cdots, \phi\left(A_{k}\right)\right) d \sigma$.

Note. Baire functions of measuroids can be defined by first taking Baire functions of measurable functions. This can be done by taking polynomials, and then almost everywhere limits. Thus, $f\left(A_{1}, \cdots, A_{k}\right)$ has an invariant meaning with respect to $A$. Further, the integral has a meaning, obtainable by first integrating a representative measurable function; and this is again invariant (i.e. independent of how $\mathbf{A}$ arose as a measuroid algebra, but depending only on the value of $\sigma$ at idempotents of $\mathbf{A}$ ). Facts and conventions of this nature will hereafter be used without further explanation.

The following two lemmas are common knowledge and are included for reference, together with a brief indication of proof.

Lemma 1. Necessary and sufficient for $m: X \rightarrow(\mathbf{A}, \sigma) \approx m^{\prime}: X \rightarrow\left(\mathbf{A}^{\prime}, \sigma^{\prime}\right)$ is that, for each Baire set $\Delta$ in Euclidean $k$-space, and each $x_{1}, \cdots, x_{k} \in X$, $\sigma\left(\left\{s \mid\left(m\left(x_{1}\right)(s), \cdots, m\left(x_{k}\right)(s)\right) \in \Delta\right\}\right)$

$$
=\sigma^{\prime}\left(\left\{s^{\prime} \mid\left(m^{\prime}\left(x_{1}\right)\left(s^{\prime}\right), \cdots, m^{\prime}\left(x_{k}\right)\left(s^{\prime}\right)\right) \in \Delta\right\}\right) .
$$

Proof. Necessity is shown by choosing the function $f$ in Definition 3 to be the characteristic function of $\Delta$. For sufficiency, $\phi$ must be constructed. This is done by first defining it on the characteristic function of $\left\{s \mid\left(m\left(x_{1}\right)(s), \cdots, m\left(x_{k}\right)(s)\right) \in \Delta\right\} ;$ this is sent to the characteristic function of $\left\{s^{\prime} \mid\left(m^{\prime}\left(x_{1}\right)\left(s^{\prime}\right), \cdots, m^{\prime}\left(x_{k}\right)\left(s^{\prime}\right)\right) \in \Delta\right\}$. This correspondence sends $\sigma$ to $\sigma^{\prime}$, and extends uniquely to an algebraic isomorphism from $\mathbf{A}$ onto $\mathbf{A}^{\prime}$.

LEMмA 2. $\bar{m} \sim \bar{m}^{\prime}$ if and only if, for every $\mathbf{A}^{\prime}$-suitable measure $\sigma^{\prime}$ there is an A-suitable measure $\sigma$ such that $(\bar{m}, \sigma) \approx\left(\bar{m}^{\prime}, \sigma^{\prime}\right)$.

Proof. Necessity is shown by defining $\sigma(T)=\int \phi\left(\chi_{T}\right) d \sigma^{\prime}$, when $\chi_{T}$ is the characteristic function of $T$. Since $\phi$ induces a lattice-isomorphism between the idempotents of $\mathbf{A}$ and those of $\mathbf{A}^{\prime}, \sigma^{\prime}$ is again a measure.

Sufficiency follows from Lemma 1. 
Hereafter, let the domain $X$ of our distributions and predistributions be a real Hilbert space. In the ensuing definitions, when an adjective is used to describe a property of a predistribution, the same adjective will be used for any distribution arising from that predistribution.

Definition 4. $\bar{m}$ will be called quasi-invariant if, for each $y \in X$, the new predistribution $\bar{m}_{y}$ obtained by setting $\bar{m}_{y}(x)=\bar{m}(x)+(x, y)$ is equivalent to $\bar{m}$.

REMARK 1 . If $m$ is a quasi-invariant distribution, and $X_{0}$ is a $k$-dimensional linear subspace of $X$, then $m \mid X_{0}$ induces a measure on the Baire sets of Euclidean $n$-space, as follows: choose a basis $x_{1}, \cdots, x_{k}$ for $X_{0}$ and a coordinate system in $n$-space, and assign to the Baire set $\Delta$ in $n$-space the measure

$$
\sigma\left(\left\{s \mid\left(m\left(x_{1}\right)(s), \cdots, m\left(x_{n}\right)(s)\right) \in \Delta\right\}\right) .
$$

Quasi-invariance of $m$ implies quasi-invariance of this $n$-dimensional measure under translation. Thus, by a well-known theorem (see [3]), the measure is equivalent to $n$-dimensional Lebesgue measure.

Definition 5. $\bar{m}$ will be called ergodic if it is quasi-invariant, and if every $A \in \mathrm{A}$ for which $\phi_{y}(A)=A$ for all $y$ ( $\phi_{y}$ being the isomorphism in the weak equivalence between $\bar{m}$ and $\bar{m}_{y}$ ) is a constant.

Definition 6. A distribution $m$ is called Gaussian if, for each $x \in X, m(x)$ has a Gaussian "distribution function" in the sense of probability theory (any nonnegative variance, any mean; variance 0 means $m(x)$ is constant, and will also be permitted).

Definition 7. $m$ is called normal with variance $c$ if it is Gaussian, and $\int m(x) m(y) d \sigma=c(x, y)$.

Definition 8. $m$ will be called completely factorable if there is an orthonormal basis $\left\{x_{j}\right\}$ for $X$ such that the $m\left(x_{j}\right)$ are independent random variables, in the sense of probability theory. It was shown in [5] that a completely factorable quasi-invariant distribution is ergodic.

\section{Examples.}

LEMмA 3. Let $\sigma_{1}, \sigma_{2}$ be mutually singular probability measures on $\mathrm{s}$. Let $\tau(T)=(1 / 2)\left(\sigma_{1}(T)+\sigma_{2}(T)\right)$. Let $\mathbf{A}_{1}, \mathbf{A}_{2}, \mathbf{B}$ be the measuroid algebras obtained from $\sigma_{1}, \sigma_{2}, \tau$ respectively. Let $\pi_{j}$ be the map which assigns to an element of $\mathbf{B}$ its coarser equivalence class in $\mathbf{A}_{j}$. Let $\pi$ be the map $A \rightarrow \pi_{1}(A) \oplus \pi_{2}(A) \in \mathbf{A}_{1} \oplus \mathbf{A}_{2}$.

(1) $\pi$ is an isomorphism from $\mathbf{B}$ onto $\mathbf{A}_{1} \oplus \mathbf{A}_{2}$.

(2) The subsets $\mathbf{S}_{j}$ of $\mathbf{A}_{j}$ are both dense if and only if $\pi^{-1}\left(\mathbf{S}_{1} \oplus \mathbf{S}_{2}\right)$ is dense in $\mathrm{B}$.

Proof. By mutual singularity, there are sets $T_{1}, T_{2}$ in $S$ such that $\sigma_{i}\left(T_{j}\right)$ $=\delta_{i j}, T_{1} \cap T_{2}=\varnothing, T_{1} \cup T_{2}=S$. Let $P_{j}$ be the $\tau$-equivalence class of the characteristic function of $T_{j}$. Then $P_{1}$ and $P_{2}$ are disjoint idempotents whose sum is the identity, and $\pi_{j}$ is one to one from $\mathbf{B} P_{j}$ onto $\mathbf{A}_{j}$. Since $\pi_{j}$ is a homomorphism, this shows that $\pi$ is an isomorphism. Since $\tau$-almost everywhere convergence holds for $\mathcal{S}$-measurable functions if and only if $\sigma_{j}$-convergence holds for both $\sigma_{1}$ and $\sigma_{2}$, (2) now follows. 
We retain this notation in the following two corollaries.

Corollary 1. Let $\bar{n}$ be a linear map from $X$ to B. Then $\pi_{j} \circ \bar{n}, j=1,2$, are both predistributions if and only if $\tilde{n}$ is a predistribution.

Proof. The set of polynomials in the $\bar{n}(x)$ has as its image under $\pi$ precisely the direct sum of the sets of polynomials in the $\pi_{1} \circ \bar{n}(x)$ and the $\pi_{2} \circ \bar{n}(x)$, so we can apply (2) of Lemma 3.

CoROllaRY 2. If $\bar{n}$ is a predistribution, and $\pi_{1} \circ \bar{n}, \pi_{2} \circ \bar{n}$ are quasi-invariant, then so is $\bar{n}$.

Proof. For $y \in X$, let $\phi_{j}$ be the automorphism of $\mathbf{A}_{j}$ sending $\pi_{j} \circ \bar{n}(x)$ to $\pi_{j} \circ \bar{n}(x)+(x, y)$. Then

$$
A \rightarrow \pi^{-1}\left(\phi_{1}\left(\pi_{1}(A)\right) \oplus \phi_{2}\left(\pi_{2}(A)\right)\right)
$$

is an automorphism of $\mathbf{B}$ sending $\bar{n}(x)$ to $\bar{n}(x)+(x, y)$.

Now, let $\Lambda$ be the real line, $S$ the set of all functions from an index set $J$ to $\Lambda$. To avoid fuss we make $J$ countable, although this is not essential. Let $\delta$ be the Borel field of sets generated by those of the form

$$
T=\left\{s \mid s\left(j_{1}\right) \in \Delta_{1}, \cdots, s\left(j_{k}\right) \in \Delta_{k}\right\},
$$

$\Delta_{1}, \cdots, \Delta_{k}$ being Baire subsets of $\Lambda$. Let $\sigma^{0}$ be a nonnegative probability measure on the Baire subsets of $\Lambda$, and let $\sigma$ be the measure induced on $\delta$ by setting $\sigma(T)=\sigma^{0}\left(\Delta_{1}\right) \cdots \sigma^{0}\left(\Delta_{k}\right)$. Assume $\int \lambda d \sigma^{0}(\lambda)=0$, and $\int \lambda^{2} d \sigma^{0}(\lambda)=c$, $c$ being a fixed positive number. Let $\lambda_{j}(s)=s(j)$, so that $\lambda_{j}$ is, for each $j \in J$, an $\delta$-measurable function, mean 0 , variance $c$ (with respect to $\sigma$ measure), and $\lambda_{j}$ is independent of $\lambda_{j^{\prime}}$ for $j \neq j^{\prime}$. We also use $\lambda_{j}$ to denote the corresponding measuroid, so that $\left\{c^{-1 / 2} \lambda_{j} \mid j \in J\right\}$ is an orthonormal set in $\mathscr{L}_{2}(\sigma)$.

Now let $X$ be a real Hilbert space with an orthonormal basis $\left\{x_{j} \mid j \in J\right\}$, and set $m(x)=\sum_{j \in J}\left(x, x_{j}\right) \lambda_{j}$. The sum converges in $\mathscr{L}_{2}(\sigma)$, and $m$ is thus a distribution, with $\int m(x) m(y) d \sigma=c(x, y)$.

EXAMPLE 1. Takes as $\sigma_{c}^{0}$ the measure on $\Lambda$ for which

$$
\frac{d \sigma_{c}^{0}(\lambda)}{d \lambda}=\frac{1}{(2 \pi c)^{1 / 2}} e^{-\lambda^{2} / 2 c} .
$$

Then $\sigma_{c}$ is the normal distribution with variance $c$. If $c \neq d$, then, by [1], $\sigma_{c}$ and $\sigma_{d}$ are mutually singular. Let $\tau=\left(\sigma_{c}+\sigma_{d}\right) / 2$, as in Lemma 3. Then the distributions $m_{c}, m_{d}$ obtained from $\sigma_{c}$ and $\sigma_{d}$ as above give a distribution $m(x)=\pi^{-1}\left(m_{c}(x) \oplus m_{d}(x)\right)$ as in Lemma 3. $m$ is quasi-invariant, but clearly not ergodic, since the automorphism sending $m(x)$ to $m(x)+(x, y)$ leaves the projections $P_{1}$ and $P_{2}$ invariant (cf. the proof of Lemma 3 ). One can see directly that for no Gaussian $n$ is $n \sim m$, since, by [1], $\tau$ would then have to be either singular to $\sigma_{c}$ or equivalent to it. 
Example 2. The notation immediately preceding Example 1 is retained, with $c=1$. Suppose the completely factorable $m$ constructed there is quasiinvariant, and suppose $m \sim m^{\prime}$ for some Gaussian distribution $m^{\prime}$. By Lemma 1 , there is a measure $\sigma_{1}$ on $S$ equivalent to $\sigma$ such that the distribution $m_{1}$ gotten by combining the predistribution of $m$ with the measure $\sigma_{1}$ is strongly equivalent to $m^{\prime}$.

Lemma 4. The map $x \rightarrow \int m_{1}(x) d \sigma_{1}$ is continuous.

Proof. Suppose $x^{(k)} \rightarrow 0$. Then $m\left(x^{(k)}\right) \rightarrow 0$ in $\mathfrak{L}_{2}(\sigma)$. Thus, for some subsequence $\left\{k_{j}\right\}$ we have $m\left(x^{\left(k_{j}\right)}\right) \rightarrow 0$ almost everywhere. So $m_{1}\left(x^{\left(k_{j}\right)}\right) \rightarrow 0$ almost everywhere, since $m$ and $m_{1}$ come from the same predistribution. In [1, Lemma 6], it is shown that then $\int m_{1}\left(x^{\left(k_{j}\right)}\right) d \sigma_{1} \rightarrow 0$. So there is some $y \in X$ such that $\int m_{1}(x) d \sigma_{1}=(x, y)$.

Lemma 5. The map $x \rightarrow m_{1}(x)$ is continuous from $X$ to $\mathfrak{L}_{2}\left(\sigma_{1}\right)$.

Proof. The map $x \rightarrow m(x)-(x, y)$ is continuous. But $m_{1}(x)-(x, y)$ is Gaussian, mean zero, so that convergence of $x$ implies almost everywhere convergence of $m_{1}(x)-(x, y)$ (see proof of Lemma 4), which implies $\mathfrak{L}_{2}\left(\sigma_{1}\right)$ convergence of $m_{1}(x)-(x, y)$, as shown in the discussion of Example 1 in [5, p. 14]. Thus, the map $x \rightarrow\left(m_{1}(x)-(x, y)\right)+(x, y)=m_{1}(x)$ is continuous.

Now let $m_{0}(x)=m_{1}(x)-(x, y)$. Then $m_{0}$ is Gaussian, mean zero, and since $m_{1}$ is quasi-invariant we have $m_{0} \sim m_{1} \sim m$.

Leмma 6. Let $f_{l}^{k}, k=1,2, \cdots ; l=1, \cdots, l(k)$ be identically distributed, nonconstant, independent measurable functions on the probability space $(R, R, \rho)$. Let $a_{l}^{k}, c^{k}$ be numbers such that $\sum_{l} a_{l}^{k} f_{l}^{k}+c^{k} \rightarrow 0$ in measure as $k \rightarrow \infty$. Then $\sum_{l} a_{l}^{k} f^{k}+c^{k} \rightarrow 0$.

Proof. Since the $f_{l}^{k}$ are nonconstant there are numbers $\gamma>0, \delta>0$ and $\beta$ such that for all $k, l$ we have

$$
\rho\left\{r \mid f_{l}^{k}(r)>\beta+\gamma\right\} \text { and } \rho\left\{r \mid f_{l}^{k}(r)<\beta\right\} \text { are both } \geqq \delta .
$$

Now, $\sum_{l} a_{l}^{k} f_{l}^{k}+c_{l}^{k}$ can be written $a_{l_{0}}^{k} f_{l_{0}}^{k}+g_{l_{0}}^{k}$, where $g_{l_{0}}^{k}$ and $f_{l_{0}}^{k}$ are independent. Then simple considerations of independence show that if $a_{l_{0}}^{k} f_{l_{0}}^{k}+g_{t_{0}}^{k}$ fluctuates by less than $\left|a_{\imath}^{k} \gamma\right|$ on a set, this set must have measure $<1-\delta$. Thus $a_{l_{0}}^{k} \rightarrow 0$ uniformly on $l_{0}$.

Now, Remark 3 in [2, p. 134] gives conditions for convergence to 0 . Condition (1) there translates into

$$
\sum_{l} \rho\left\{\left|a_{l}^{k} f_{l}^{k}\right|>\epsilon\right\}+d^{k} \rightarrow 0 \quad \text { for each } \epsilon>0
$$

where 


$$
d^{k}=\rho\left\{\left|c^{k}\right|>\epsilon\right\}=0
$$

if $\left|c^{k}\right|>\epsilon$, if $\left|c^{k}\right| \leqq \epsilon$.

Then $c^{k} \rightarrow 0$. Condition (2) gives

$$
\sum a_{l}^{k} \int_{\Delta_{l}^{k}} f_{l}^{k} d \rho \rightarrow 0
$$

But

$$
\int_{\Delta_{l}^{k}} f_{l}^{k} d \rho \rightarrow 1\left(\text { where } \Delta_{l}^{k}=\left\{r \mid a_{l}^{k} f_{l}^{k}(r)<\epsilon\right\}\right)
$$

uniformly in $l$, since $a_{l}^{k} \rightarrow 0$ uniformly in $l$. Thus $\sum_{l} a_{l}^{k} \rightarrow 0$.

COROLlaRY. Let $h_{k}$ be a finite sum $\sum v_{i j} g_{i} g_{j}+w$, for certain measurable functions $g_{j}$ with jointly Gaussian distributions of mean 0 and certain numbers $v_{i j}, w$ (actually depending on $k$, of course, but this is suppressed in the notation). Let $h_{k} \rightarrow 0$ in measure. Then $\int h_{k} d \rho \rightarrow 0$.

Proof. Choose an orthonormal basis for the linear subspace of $\mathscr{L}_{2}(\rho)$ spanned by the $g_{j}$. Then the $g_{j}$ are finite linear combinations of these basis elements, and the effect on the expression for $h_{k}$ is that one can assume without loss of generality that the $g_{j}$ are orthonormal. And, of course, one can let $v_{i j}=v_{j i}$. Now choose an orthogonal matrix $U=\left(u_{i j}\right)$ such that $U\left(v_{i j}\right) U^{t}$ is diagonal. Then, letting $\bar{g}_{i}=\sum u_{i j} g_{j}$, we get $h_{k}$ in the form $\sum a_{j} \bar{g}_{j}^{2}+w$.

Now Lemma 6 can be applied, since $\int h_{k} d \rho$ is exactly $\sum a_{j}+w$.

Next, let $\left\{c_{i j} \mid i, j \in J\right\}$ be a square-summable set of real numbers, and consider the sum

$$
\sum_{i} c_{i i}\left(m\left(x_{i}\right)^{2}-1\right)+\sum_{i \neq j} c_{i j} m\left(x_{i}\right) m\left(x_{j}\right) .
$$

This converges in $\mathscr{L}_{2}(\sigma)$, since

$$
\left\{m\left(x_{i}\right)^{2}-1 \mid i \in J\right\} \cup\left\{m\left(x_{i}\right) m\left(x_{j}\right) \mid i \neq j ; i, j \in J\right\}
$$

forms an orthogonal set in $\mathscr{L}_{2}(\sigma)$ : not normal, indeed, but with exactly two nonzero norms occurring, which is equally good for our purpose. Thus there is an increasing sequence $J_{k}$ of finite subsets of $J$, with $U_{k} J_{k}=J$, and such that the partial sums

$$
s_{k}=\sum_{j \in J_{k}} c_{j j}\left(m\left(x_{j}\right)^{2}-1\right)+\sum_{i, j \in J_{k}} c_{i j} m\left(x_{i}\right) m\left(x_{j}\right)
$$

are a Cauchy sequence in $\sigma$-measure, and therefore also in $\sigma_{1}$-measure. By the corollary to Lemma 6 , we then have $\int\left(s_{k}-s_{l}\right) d \sigma_{1} \rightarrow 0$, so that 


$$
\sum_{i \in J_{k}} c_{i i}\left(\int m_{0}\left(x_{i}\right)^{2} d \sigma_{1}-1\right)+\sum_{i, j \in J_{k} ; i \neq j} c_{i j} \int m_{0}\left(x_{i}\right) m_{0}\left(x_{j}\right) d \sigma,
$$

converges as $k \rightarrow \infty$. Since the signs of the $c_{i j}$ may be changed at will, the series

$$
\sum_{i \in J} c_{i i}\left(\int m_{0}\left(x_{i}\right)^{2} d \sigma_{1}-1\right)+\sum_{i, j \in J} c_{i j} \int m_{0}\left(x_{i}\right) m_{0}\left(x_{j}\right) d \sigma_{1}
$$

actually converges unconditionally. Then

$$
\sum_{i \in J}\left|\int m_{0}\left(x_{i}\right)^{2} d \sigma_{1}-1\right|^{2}+\sum_{i, j \in J}\left|\int m_{0}\left(x_{i}\right) m_{0}\left(x_{j}\right) d \sigma_{1}\right|^{2}<\infty .
$$

This says that the operator $m_{0}$ from $X$ to $\mathscr{L}_{2}\left(\sigma_{1}\right)$ has the property that $m_{0}^{*} m_{0}-I$ is a Hilbert-Schmidt operator. By [5], this implies that $m_{0} \sim n$, where $n$ is a normal distribution on $X$ with variance 1 .

Then $m \sim n$, and so there is a measure $\tau$ on $\delta$ which is equivalent to $\sigma$, and for which the coordinate functions $\lambda_{j}$ are independent, mean 0 , variance 1 , with Gaussian distribution functions. But a simple consequence of Kakutani's criterion for equivalence of infinite product measures says that can happen only if $\lambda_{j}$ has the same distribution with respect to $\sigma$, i.e. if $\tau=\sigma$.

All that remains is to construct $\sigma^{0}$ on $\Lambda$ such that $\int d \sigma^{0}=1, \int \lambda d \sigma^{0}(\lambda)=0$, $\int \lambda^{2} d \sigma^{0}(\lambda)=1$, and such that the $\sigma$ obtained from it is quasi-invariant. A necessary condition (cf. Remark 1 ) is that $d \sigma^{0}(\lambda)=g^{2}(\lambda) d \lambda$ for some positive Lebesgue square-integrable function $g$, with

$$
\begin{aligned}
& \int g^{2}(\lambda) d \lambda=1, \\
& \int \lambda g^{2}(\lambda) d \lambda=0, \\
& \int \lambda^{2} g^{2}(\lambda) d \lambda=1 .
\end{aligned}
$$

(2) can be guaranteed by making $g$ even, together with (3). Let $G(\mu)$ $=(2 \pi)^{-1 / 2} \int e^{i \lambda \mu} g(\mu) d \mu$. Let us assume further that

$$
\int \mu^{2}|G(\mu)|^{2} d \mu<\infty
$$

We now find the condition for quasi-invariance to be (using Lemma 2 and Kakutani's criterion)

$$
\prod_{j \in J}\left(\int g\left(\lambda+c_{j}\right) g(\lambda) d \lambda\right)>0 \quad \text { if } \sum\left|c_{j}\right|^{2}<\infty .
$$


Plancherel's theorem says

$$
\int g(\lambda+c) g(\lambda) d \lambda=\int e^{i \mu c}\left|G^{2}(\mu)\right| d \mu
$$

so $\left(^{*}\right)$ becomes

$$
\prod_{j \in J} \int e^{i \mu c_{j}}\left|G^{2}(\mu)\right| d \mu>0 \quad \text { if } \sum_{j}\left|c_{j}\right|^{2}<\infty .
$$

Note that each factor is positive. We rewrite the product as

$$
\prod_{j}\left(1-2 \int \sin ^{2}\left(\frac{\mu c_{j}}{2}\right)|G(\mu)|^{2} d \mu\right) .
$$

Now,

$$
2 \int \sin ^{2}\left(\frac{\mu c}{2}\right)|G(\mu)|^{2} d \mu \leqq \frac{c^{2}}{2} \int \mu^{2}|G(\mu)|^{2} d \mu,
$$

so the infinite product converges to a nonzero limit if $\sum\left|c_{j}\right|^{2}<\infty$.

\section{REFERENCES}

1. J. Feldman, Equivalence and perpendicularity of Gaussian processes, Pacific J. Math. vol. 8 (1958) pp. 699-708.

2. B. V. Gnedenko and A. N. Kolmogorov, Limit distributions for sums of independent random variables (translated by K. L. Chung), Cambridge, Addison-Wesley, 1954.

3. G. W. Mackey, Induced representations of locally compact groups. I, Ann. of Math. vol. 55 (1952) pp. 101-139.

4. I. E. Segal, Equivalence of measure spaces, Amer. J. Math. vol. 73 (1951) pp. 275-313.

5. - Distributions in Hilbert space and canonical systems of operators, Trans. Amer. Math. Soc. vol. 88 (1958) pp. 12-41.

6. - Foundations of the theory of dynamical systems of infinitely many variables, Mat.Fys. Medd. Danske. Vid. Selsk. vol. 31 (1959) pp. 1-38.

7. S. Kakutani, On equivalence of infinite product measures, Ann. of Math. vol. 49 (1948) pp. 214-224.

University of California,

Berkeley, California 